\title{
DISLIPIDEMIAS E ALGUNS FATORES DE RISCO ASSOCIADOS EM UMA POPULAÇÃO PERIFÉRICA DA REGIÃO METROPOLITANA DE SÃO PAULO, SP - BRASIL. UM ESTUDO PILOTO
}

\author{
Ignez Salas Martins* \\ Leda Teixeira Coelho* \\ Isaura Maria de Souza Matos* \\ Rosa Nilda Mazzilli* \\ Marlene Trigo* \\ Donald Wilson*
}

\begin{abstract}
MARTINS, I.S. et al. Dislipidemias e alguns fatores de risco associados em uma população periférica da Região Metropolitana de São Paulo, SP - Brasil. Um estudo piloto. Rev. Saúde públ., S. Paulo, 23: $236-43,1989$.

RESUMO: Realizou-se levantamento da prevalência de dislipidemias e alguns fatores de risco (hipertensão, obesidade e alcoolismo), em uma população típica da periferia do Município de São Paulo, SP (Brasil). A prevalência de um ou mais fatores de risco foi maior do que $50 \%$ em todos os grupos etários. No que concerne as dislipidemias propriamente ditas, foi de aproximadamente $49 \%, 58 \%$ e $57 \%$ nos gru: pos etários de 20 a 39 anos, 40 a 59 anos e 60 anos e mais, respectivamente. A dislipidemia mais freqüente foi devido a baixos niveis de HDL-colesterol, principalmente nos mais jovens, entre 29 e 39 anos. Nos outros dois grupos etários houve prevalência maior de "outras dislipidemias". A obesidade isolada ou associada foi o fator de risco mais relevante na população estudada. Levanta-se a hipótese de que as dislipidemias podem constituir-se em grave problema de Saúde Pública nas populações periféricas da cidade de São Paulo.
\end{abstract}

DESCRITORES: Hiperlipidemia, incidência. Lipoproteinas do HDL-colesterol, sangue. Colesterol, sangue. Triglicérides, sangue. Risco.

\section{INTRODUÇÃO}

O presente trabalho faz parte do Projeto de Integração Docente Assistencial de Itapecirica da Serra, que tem por finalidade a integração dos serviços de atenção primária, secundária, terciária e quaternária de saúde, tendo em vista a prevenção e o controle de doenças importantes do ponto de vista da Saúde Pública, no Município de Cotia, Região Metropolitana de São Paulo.

Há muito suspeita-se que as doenças cardiovasculares ateroscleróticas, isquêmicas do coração e hipertensivas sejam problema de Saúde Pública no Brasil, à medida em que parecem existir fatores de risco dessas doenças, disseminados na população de todos os níveis sócioeconômicos.

As dislipidemias são causas fundamentais de doenças coronarianas ateroscleróticas ${ }^{1,7,8}$, 10,16,30,32,34. A prevenção dessas morbidades e dos fatores de risco, que se apresentem como determinantes ou associados às mesmas, tem aumentado consideravelmente a esperança de vida de grupos populacionais em fase produtiva, principalmente de paises desenvolvidos, onde há muito se realizam pesquisas epidemiológicas, pelos estudos longitudinais e transversais, seguidas da aplicação de programas de intervenção ${ }^{15,19,21}$.

A observação do cotidiano de populaçð̃es pobres de diferentes localidades da Região Metropolitana de São Paulo permite levantar a hipótese da existência de doenças cardiovasculares ateroscleróticas, isquêmicas do coração e hipertensivas como problema de Saúde Pública, à medida em que fatores de risco, tais como, tabagismo, alcoolismo, obesidade e estresse decorrente do pauperismo fazem-se presentes, necessitando ser avaliados por meio de pesquisas epidemiológicas. Por isso optou-se pela realização de pesquisa-piloto, de caráter exploratório, numa comunidade típica da Região Metropolitana. Neste sentido, foi feito um estudo epidemiologico piloto, no Distrito de Caucaia do Alto, Município de Cotia. O estudo focalizou algumas doenças não-transmissíveis, tais como dislipidemias, obesidade, hipertensão, diabetes melito, coronariopatias e respectivos fatores de risco. Como parte dele, o presente trabalho analisa a prevalência de dislipidemias e os seguintes fatores de risco: obesidade, hipertensão

* Departamento de Nutrição da Faculdade de Saúde Pública da Universidade de Săo Paulo - Av. Dr. Arnaldo, 715 01255 - São Paulo, SP - Brasil. 
e etilismo; procura, também, caracterizar a intensidade com que cada um deles se encontra associado às dislipidemias, com o intuíto de se conhecer a gravidade com que se apresentam como fatores de risco das mesmas. A obesidade e o etilismo são fatores de risco de dislipidemias já conhecidos, enquanto que a hipertensão tem sido apontada como tal em pesquisas mais recentes 22,31 .

O Distrito de Caucaia pode ser considerado típico da periferia da Região Metropolitana de São Paulo, quer seja pelo seu despojamento de recursos sanitários, transporte e moradia, quer seja pela forma com que sua população se insere no mercado de trabalho: trabalhadores (principalmente não qualificados), pequenos comerciantes e funcionários públicos.

\section{MATERIAL E MÉTODO}

\section{Amostra}

Após cadastramento da área urbana o Distrito de Caucaia do Alto, foram escolhidas ao acaso 154 famílias, das quais foram selecionados todos os indivíduos acima de 20 anos de idade, de ambos os sexos, totalizando 279 pessoas. Esta amostra representativa da população foi submetida a exames clínico e bioquímico.

\section{Exame Clínico}

O exame clínico constou de medidas antropométricas (peso, altura), dados anamnésticos constando de antecedentes familiares e pessoais de diabetes melito, doenças cardiovasculares ateroscleróticas, coronariopatias e respectivos fatores de risco. Além dos dados anamnésticos foi realizado exame físico geral e especial.

A pressão arterial foi medida com esfigmomanômetro de mercúrio, sendo considerada normal até $140 \mathrm{~mm}$ de $\mathrm{Hg}$ para a sistólica de 90 $\mathrm{mm}$ de $\mathrm{Hg}$ para diastólica. Foram feitas três tomadas consecutivas de pressão no braço direito do indivíduo sentado, sendo a média, dessas medidas, o valor considerado.

Foram realizados, em amostras de sangue colhida em jejum, os seguintes exames bioquímicos: colesterol total, pelo método enzimático; HDL-colesterol, pelo método enzimático após precipitação pelo ácido fosfotungstico na presença de ions de $\mathrm{Mg}$; triglicérides, pelo método enzimático.

Os valores considerados normais para os índices bioquímicos foram os seguintes: colesterol total, até $220 \mathrm{mg} / \mathrm{dl}$; HDL-colesterol, níveis acima de $45 \mathrm{mg} / \mathrm{dl}$ para mulheres e $35 \mathrm{mg} / \mathrm{dl}$ para homens; triglicérides, até $140 \mathrm{mg} / \mathrm{dl}^{*}$.

A obesidade foi definida pela relação peso e altura tendo sido utilizada a tabela de peso adotada pela Metropolitan Life Insurance $\mathrm{Co}^{2}$. Foram considerados obesos os indivíduos com peso corporal acima do limite superior do intervalo de normalidade para uma determinada altura.

Foram considerados etilistas os indivíduos que declararam ingerir álcool diariamente ou regularmente (mais de uma vez por semana).

\section{RESULTADOS}

A Tabela 1 mostra grande prevalência, cerca de $74 \%$ dos fatores de risco de dislipidemia abordados (obesidade, hipertensão e alcoolismo), isolados ou associados. Pode-se constatar

TABELA 1

Presença de fatores de risco de dislipidemias segundo idade. Distrito de Caucaia do Alto, Município de Cotia, Área Metropolitana de São Paulo - SP (Brasil), 1987.

\begin{tabular}{ccccc}
\hline \multirow{2}{*}{$\begin{array}{c}\text { Idade } \\
\text { (anos) }\end{array}$} & \multicolumn{3}{c}{ Fatores de Risco } & Total \\
\cline { 2 - 4 } & Isentos & 1 fator & 2 ou + fatores & \\
\hline $20 \vdash 39$ & $51(35,9 \%)$ & $78(54,8 \%)$ & $13(9,3 \%)$ & $142(100 \%)$ \\
$40 \vdash 59$ & $11(10,3 \%)$ & $41(38,3 \%)$ & $55(51,4 \%)$ & $107(100 \%)$ \\
$60++$ & $1(3,3 \%)$ & $12(40,0 \%)$ & $17(56,7 \%)$ & $30(100 \%)$ \\
\hline Total & $63(26,2 \%)$ & $131(46,9 \%)$ & $85(26,9 \%)$ & $279(100 \%)$ \\
\hline
\end{tabular}

\footnotetext{
* LABTEST - Colesterol total e fraçðes - significado clínico e análise dos fatores de risco. Atualizaçðes Labtest $N .^{\circ} 1$.
} 
que em todos os grupos etários considerados, mais de $50 \%$ dos indivíduos apresentaram algum fator de risco. Nos mais jovens, a prevalência de um fator de risco foi de aproximadamente $55 \%$ e de dois ou mais fatores, um pouco mais de 9\%. Por sua vez, nas idades mais avançadas há predominância de dois ou mais fatores de risco.

Tais fatores, dentro da história natural das doenças, constituem-se em estímulos ao desencadeamento da disfunção orgânica. Assim, é de se esperar alta prevalência de dislipidemias na população. A Tabela 2 não só é reveladora desse fato mas mostra alta prevalência, cerca de $49 \%$, no grupo etário de 20 e 39 anos. Nos indivíduos com idades entre 40 e 59 anos, a prevalência foi de, aproximadamente, $58 \%$ e nos mais velhos $57 \%$.
A prevalência de dislipidemias, segundo grupo etário, sexo e fatores de risco, é apresentada na Tabela 3. Pode-se constatar que a hipertensão, alcoolismo e obesidade predominam no sexo masculino, enquanto que nas mulheres a obesidade isolada ou associada à hipertensão estiveram presentes em maior proporção, predominando esta última, como é de se esperar, no grupo etário acima de 40 anos. No cômputo geral, cerca de $47 \%$ das dislipidemias ocorreram no grupo etário mais jovem: $17,4 \%$ nos homens e $29,5 \%$ nas mulheres.

No grupo etário de 40 a 59 anos, a prevalência foi de $22,1 \%$ e $18,8 \%$ nos homens e nas mulheres, respectivamente, e nos mais velhos, pouco presentes na amostra, foi de $4,6 \%$ e $7 \%$.

A Tabela 4, por sua vez, aponta que quase $56 \%$ dos casos, no grupo etário de 20 a 39 anos,

TABELA 2

Presença de dislipidemia, segundo idade. Distrito de Caucaia do Alto, Município de Cotia, Área Metropolitana de São Paulo - SP (Brasil), 1987.

\begin{tabular}{llll}
\hline \multirow{2}{*}{ Idade } & \multicolumn{2}{c}{ Dislipidemia } & Total \\
\cline { 2 - 4 } & Presença & Ausência & $142(100 \%)$ \\
$29 \vdash 39$ & $70(49,3 \%)$ & $72(50,7 \%)$ & $107(100 \%)$ \\
$40 \vdash 59$ & $62(57,9 \%)$ & $45(42,1 \%)$ & $30(100 \%)$ \\
$60 \mathrm{e}+$ & $17(56,6 \%)$ & $13(43,3 \%)$ & $279(100 \%)$ \\
\hline Total & $149(53,4 \%)$ & $130(46,6 \%)$ & \\
\hline
\end{tabular}

\section{TABELA 3}

Prevalência de dislipidemias, de acordo com fatores de risco, segundo sexo e idade. distrito de Caucaia do Alto, Município de Cotia, Área Metropolitana de Sæo Paulo (SP), Brasil, 1987.

\begin{tabular}{|c|c|c|c|c|c|c|c|}
\hline \multirow[b]{2}{*}{$\begin{array}{c}\text { Idade e } \\
\text { sexo }\end{array}$} & \multicolumn{6}{|c|}{ Dislipidemias } & \multirow[b]{2}{*}{ Total } \\
\hline & Ausência & Hipertensão & Etilismo & Obesidade & $\begin{array}{c}\text { Obesidade } \\
+ \\
\text { Hipertensăo }\end{array}$ & $\begin{array}{l}\text { Obesidade + } \\
\text { etilismo com ou } \\
\text { sem Hipertensão }\end{array}$ & \\
\hline
\end{tabular}

Homens

\begin{tabular}{|c|c|c|c|c|c|c|c|c|c|c|c|c|c|c|}
\hline $\begin{array}{l}20-39 \\
40-59 \\
60 \text { e mais }\end{array}$ & 1 & $\frac{(6,2 \%)}{(12,5 \%)}$ & $\begin{array}{l}7 \\
3\end{array}$ & $\begin{array}{l}(58,3 \%) \\
(25,0 \%) \\
-\end{array}$ & $\begin{array}{r}10 \\
6\end{array}$ & $\begin{array}{l}(41,4 \%) \\
(27,3 \%) \\
-\end{array}$ & $\begin{array}{l}3 \\
6 \\
1\end{array}$ & $\begin{array}{r}(6,8 \%) \\
(13,6 \%) \\
(2,2 \%)\end{array}$ & $\begin{array}{l}6 \\
2\end{array}$ & $\begin{array}{r}- \\
(18,1 \%) \\
(6,1 \%)\end{array}$ & $\begin{array}{r}5 \\
12 \\
2\end{array}$ & $\begin{array}{r}(11,8 \%) \\
(60,9 \%) \\
(8,6 \%)\end{array}$ & $\begin{array}{l}26 \\
33\end{array}$ & $\begin{array}{r}(17,4 \%) \\
(22,1 \%) \\
(4,7 \%)\end{array}$ \\
\hline
\end{tabular}

\section{Mulheres}

\begin{tabular}{|c|c|c|c|c|c|c|c|}
\hline $\begin{array}{l}20-39 \\
40-59 \\
60 \text { e mais }\end{array}$ & $\begin{array}{l}(81,3 \%) \\
-\end{array}$ & $\begin{array}{c}2 \overline{(16,6 \%)} \\
-\end{array}$ & $\begin{array}{c}6(27,3 \%) \\
- \\
-\end{array}$ & $\begin{array}{rr}21 & (47,7 \%) \\
11 & (25,0 \%) \\
2 & (4,5 \%)\end{array}$ & $\begin{array}{rr}3 & (9,0 \%) \\
15 & (45,4 \%) \\
7 & (21,2 \%)\end{array}$ & $\begin{array}{ll}1 & (4,3 \%) \\
2 & (8,6 \%)\end{array}$ & $\begin{array}{lr}44 & (29,5 \%) \\
28 & (18,8 \%) \\
11 & (7,4 \%)\end{array}$ \\
\hline Total & $16(100 \%)$ & $12(100 \%)$ & $22(100 \%)$ & $44(100 \%)$ & $33(100 \%)$ & $22(100 \%)$ & $149(100 \%)$ \\
\hline
\end{tabular}


foram devido a níveis de HDL-colesterol abaixo da normalidade, enquanto que no grupo etário de 40 a 59 anos essa proporção foi de $34 \%$. Assim, $42 \%$ das dislipidemias ocorreram devido a baixos níveis dessa fração lipoprotéica. Também, nas dislipidemias restantes (cerca de $29 \%$ ) aproximadamente em $58 \%$ delas o HDLcolesterol esteve em concentração abaixo da normalidade.

A Tabela 5 esclarece melhor a prevalência do HDL-colesterol e "outras dislipidemias" Pode-se observar que, no grupo etário mais jovem, $22 \%$ deveu-se a "outras dislipidemias" e $27,5 \%$ a níveis baixos de HDL-colesterol. As proporções de HDL-colesterol baixo diminui à medida que a idade aumenta, e as de "outras dislipidemias" comportam-se de maneira inversa.

A relação, entre as dislipidemias e os fatores de risco abordados, comprovada em diferentes estudos ${ }^{1,5,7.12,23,30,32}$, é sugerida na Tabela 6 , que mostra a obesidade isolada ou associada à hipertensão e/ou ao alcoolismo, presente em mais ou menos $30 \%$ e $37 \%$ dos casos, respectivamente. $O$ restante ocorre em hipertensos $(8,1 \%)$ e etilistas $(14,8 \%)$. Pode-se, também, constatar que mais ou menos $26 \%$ dos casos caracterizados pela "ausência de risco" apresentaram baixos níveis de HDL-colesterol. Isto, provavelmente se deve ao fato de que nem todos os fatores de risco dessa dislipidemia foram considerados no presente trabalho.

Por outro lado, a "razão de prevalência" de dislipidemias, segundo os fatores de risco e as associações encontradas (Tabela 7), apontam a obesidade isolada ou associada como fator de risco mais relevante entre os abordados no presente trabalho.

\section{COMENTÁRIOS}

A prevalência de fatores de risco na população estudada foi bastante alta, estando apenas $26 \%$ dos indivíduos (Tabela 1) isentos. Conseqüentemente, a prevalência de dislipidemis (Tabelas 2 e 3) foi também alta, em todos os grupos etários. Esses resultados permitem duas suposiçóes. A primeira é a de que os padroes de referência utilizados, extraídos de estudos realizados em outros países, poderiam não ser adequados à nossa realidade. A necessidade de pesquisas, tendo em vista o estabelecimento de padrôs de referência, a partir de dados levantados em populaçoes brasileiras, é um fato ponderável. Trabalho realizado no Centro de Saúde Geraldo de Paula Souza, no Município de São $\mathrm{Paulo}^{24}$, parece confirmar os padrōes de re- 
TABELA 5

Dislipidemias decorrentes de baixo teor de HDL-colesterol sangüineo, "butras dislipidemias", e ausência de dislipidemia, segundo grupo etário. Distrito de Caucaia do Alto, Município de Cotia, Area

Metropolitana de São Paulo - SP (Brasil), 1987.

\begin{tabular}{lcccr}
\hline & & Dislipidemias & & \\
\cline { 2 - 4 } Idade & $\begin{array}{c}\text { Dislipidemia } \\
\text { Colesterol } \\
\text { devido ao HDL- } \\
\text { Colesterol }\end{array}$ & $\begin{array}{c}\text { “Outras } \\
\text { Dislipidemias" }\end{array}$ & $\begin{array}{c}\text { Ausencia de } \\
\text { Dislipidemia }\end{array}$ & Total \\
\hline $20+39$ & $39(27,5 \%)$ & $31(21,8 \%)$ & $72(50,7 \%)$ & $142(100 \%)$ \\
$40-59$ & $21(19,6 \%)$ & $41(38,3 \%)$ & $45(42,1 \%)$ & $107(100 \%)$ \\
$60+$ & $2(6,7 \%)$ & $15(50,0 \%)$ & $13(43,3 \%)$ & $30(100 \%)$ \\
\hline Total & $62(22,2 \%)$ & $87(31,2 \%)$ & $130(46,6 \%)$ & $279(100 \%)$ \\
\hline
\end{tabular}

ferência internacionais referentes a colesterolemia e trigliceridemia, à medida em que nos grupos apresentando "ausência de risco" de doenças cardiovasculares ateroscleroticas, os níveis séricos de colesterol total e triglicérides apresentaram valores coincidentes com os niveis considerados normais na literatura. A segunda suposição é a de que sendo válido os padrós adotados, a prevalência de dislipidemias e fatores de risco foi alta, permitindo que se desmistifique a idéia, muito difundida, de que os fatores de risco de doenças cardiovasculares ateroscleróticas e doença isquêmica do coração acometem, principalmente, indivíduos mais velhos, fora da idade produtiva e de classes sociais elevadas.

A dislipidemia mais prevalente foi devido a baixas taxas de HDL-colesterol (Tabela 4), principalmente nos mais jovens, diminuindo com a idade, como se a depressão dessa fração do colesterol fosse o início do processo de distúrbio de metabolismo lipídico na história natu- ral da doença. Sabe-se que o HDL-colesterol tem função protetora, removendo os ateromas das artérias. O estudu prospectivo de Framingham ${ }^{8}$ mostrou que 0 risco de se adquirir doenças cardiovasculares ateroscleróticas é maior em individuos com baixas taxas de HDLcolesterol, mesmo naqueles com niveis baixos de colesterol total. Entretanto, outro estudo da mesma natureza ${ }^{27}$, envolvendo 24 cidades britâ. nicas, sugere ser irrelevante o papel do HDLcolesterol baixo como fator de risco de doenças isquêmicas do coração ${ }^{25}$. Assim, a dislipidemia decorrente do HDL-colesterol precisa ser objeto de estudos, porque além de serem controvertidos os resultados sobre sua ação na etiologia das doenças cardiovasculares, esse distúrbio lipêmico parece ter prevalência expressiva em nosso meio. A literatura a respeito do tema tem apontado que níveis baixos de HDL-colesterol podem estar associados à desnutrição, subnutrição energética, inatividade física, distúrbios

\section{TABELA 6}

Dislipidemias, segundo fatores de risco. Distrito de Caucaia do Alto, Município de Cotia Area Metropolitana de São Paulo - SP, Brasil, 1987

\begin{tabular}{|c|c|c|c|c|c|c|c|c|}
\hline \multirow[b]{2}{*}{ Fatores de Risco } & \multicolumn{7}{|c|}{ Dislipidemias } & \multirow[b]{2}{*}{ Total } \\
\hline & Colesterol $\uparrow$ & $\begin{array}{l}\text { Triglicé- } \\
\text { rides } \uparrow\end{array}$ & $\begin{array}{c}\text { HDL: } \\
\text { Colesterol } \downarrow\end{array}$ & $\begin{array}{c}\text { Colesterol } \uparrow \\
+ \\
\text { Triglicérides } \uparrow\end{array}$ & $\begin{array}{c}\text { Triglicérides } \uparrow \\
+ \\
\text { HDL-Colesterol } \downarrow\end{array}$ & $\begin{array}{c}\text { Colesterol } \uparrow \\
+ \\
\text { HDL-Colesterol } \downarrow\end{array}$ & $\begin{array}{l}\text { Culesterol } \uparrow+1 \text { ri- } \\
\text { glicérides } \uparrow+110 L \text { - } \\
\text {-Colesterol } \downarrow\end{array}$ & \\
\hline ausência & • & - & $16(25,4 \%)$ & - & $\cdot$ & - & $\cdot$ & $16(10.7 \%)$ \\
\hline obesidade & $5(21.7 \%)$ & $5(35,7 \%)$ & $19(30,1 \%)$ & - & $10(55,6 \%)$ & $2(18.2 \%)$ & $3(21,4 \%)$ & $44(295 \%)$ \\
\hline hipertensão & $2(8,7 \%)$ & $1(7,1 \%)$ & $3(4,9 \%)$ & - & - & $1(9,1 \%)$ & $5(35,7 \%)$ & $12(8.1 \%)$ \\
\hline etilismo & $2(8,7 \%)$ & $3(21,4 \%)$ & $11(17,4 \%)$ & $3(42,9 \%)$ & $3(16,7 \%)$ & - & • & $22(14,8 \%)$ \\
\hline \multicolumn{9}{|c|}{$\begin{array}{l}\text { obesidade + etillismo } \\
\text { com ou sem }\end{array}$} \\
\hline hipertensão & $14(60,9 \%)$ & $5(35,7 \%)$ & $14(22,2 \%)$ & $4(57,1 \%)$ & $5(27,8 \%)$ & $8(72,7 \%)$ & $6(42,9 \%)$ & $55(36,9 \%)$ \\
\hline Total & $23(100 \%)$ & $14(100 \%)$ & $63(100 \%)$ & $7(100 \%)$ & $18(100 \%)$ & $11(100 \%)$ & $14(100 \%)$ & $149(100 \%)$ \\
\hline
\end{tabular}


TABELA 7

Razão cie prevalência* de dislipidemias, segundo fatores de risco, Distrito de Caucaia do Alto, Município de Cotia, Área Metropolitana de São Paulo - SP (Brasil), 1987.

\begin{tabular}{|c|c|c|c|c|c|c|}
\hline \multirow[b]{2}{*}{ Indivíduos } & \multicolumn{6}{|c|}{ Dislipidemias } \\
\hline & Ausência & Presença & Total & Po & $\mathrm{Pe}$ & $\begin{array}{l}\text { Razão de } \\
\text { Prevalência }\end{array}$ \\
\hline "Isentos" & $47(74,6 \%)$ & $16(25,4 \%)$ & $63(100 \%)$ & 0,25 & - & - \\
\hline Hipertensos & $11(47,0 \%)$ & $12(52,0 \%)$ & $23(100 \%)$ & & 0,52 & 2,08 \\
\hline Etilistas & $18(45,0 \%)$ & $22(55,0 \%)$ & $40(100 \%)$ & & 0,55 & 2,20 \\
\hline Obesos & $25(36,2 \%)$ & $44(63,8 \%)$ & $69(100 \%)$ & & 0,64 & 2,56 \\
\hline Obesos Hipertensos & $13(28,3 \%)$ & $33(71,7 \%)$ & $46(100 \%)$ & & 0,72 & 2,88 \\
\hline Obesos Etilistas & $6(35,3 \%)$ & $10(64,7 \%)$ & $16(100 \%)$ & & 0,65 & 2,60 \\
\hline Obesos Hiper tensos & & & & & & \\
\hline e Etilistas & $10(40,0 \%)$ & $12(60,0 \%)$ & $22(100 \%)$ & & 0,60 & 2,40 \\
\hline
\end{tabular}

$\mathrm{X}^{2}$ para "isentos" $\mathrm{e}$ hiper tensos $=5,53$

$X^{2}$ para "isentos" e etilistas $=9,32$

$X^{2}$ para "isentos" e obesos $=19,45$

$\mathrm{X}^{2}$ para "isentos" e obesos hipertensos $=22,98$

$\mathrm{X}^{2}$ para "isentos" e obesos etilistas $=7,66$

$\mathrm{X}^{2}$ para "isentos" $\mathrm{e}$ obesos hipertensos e etilistas $=6,40$

$\mathrm{X}=0,05$

Valor crítico $=3,84$

* Ver Lapidus e col.20 (1986)

hormonais ligados à progesterona e ao estradiol ${ }^{20,25,29,33}$. Voltando à Tabela 3, pode-se observar que $26 \%$ dos casos caracterizados pela "ausência de risco" apresentaram níveis baixos de HDL-colesterol, sendo que a maior proporção deles $(81,3 \%)$ ocorreu em mulheres jovens, o que permite a hipótese de que a vida sedentária da mulher dona de casa, aliada ao fato de possivelmente usarem contraceptivos orais, possa ser responsável por esse quadro.

O presente trabalho colabora para confirmar outros estudos ${ }^{22,31}$ que apresentam a hipertensão como fator de risco de dislipidemias, uma vez que $52 \%$ dos hipertensos apresentaram niveis lipêmicos anormais, sendo a razão de prevalência entre isentos e hipertensos igual a 2,08 e a associação $\mathrm{X}^{2}$, encontrada entre ambos, foi significante para $\alpha=0,05$.

Por outro lado, a obesidade, isolada ou associada à hipertensão e/ou ao alcoolismo, foi o fator de risco mais relevante de dislipidemias encontrado no presente trabalho, coincidindo com resultados de outras pesquisas e com trabalho anterior, realizado em uma população do Município de São Paulo 4,11,12,14,16,17,24,26,28,35.

Nos indivíduos considerados etilistas, a proporção de dislipidemias foi de aproximadamente $50 \%$, com razão de prevalência igual a 2,24 (Tabela 7). Ressalte-se que, no presente trabalho, foi considerado etilista todo indivíduo que declarou ingerir álcool diariamente ou regularmente (mais de uma vez por semana), sem se levar em conta a quantidade ingerida. Há evidências de que a ingestão de quantidades discretas de álcool produz um efeito protetor frente às dislipidemias, fato que, possivelmente, esteja influenciando os resultados do presente trabalho 3,6,9,14,17,36.

A prevalência de dislipidemias, encontrada no presente estudo piloto, seja ela decorrente de baixas taxas do HDL-colesterol ou de "outras dislipidemias", em todos os grupos etários, provavelmente seja grave problema de Saúde Pública nesta população típica estudada, da periferia da Região Metropolitana de São Paulo, podendo esse quadro se reproduzir em situações semelhantes.

\section{AGRADECIMENTOS}

À Rita de Cássia Montes, Rosário Alonso Nieto e Elaine Donizette Alvares (Nutricionistas); Elisa Tieko Okani (Farmacêutica Bioquímica) e Maria Emigdio Silvéria (Auxiliar de laboratório) do Departamento de Nutrição da Faculdade de Saúde Pública da Universidade de São Paulo, pela colaboração na coleta de dados. 
MARTINS, I.S. et al. [Lipemic disorders and some associated risk factors in a population on the outs kirts of Greater S. Paulo, SP, Brazil]. Rev. Saúde públ., S. Paulo, 23: 236-43, 1989.

ABSTRACT: A survey of the prevalence of lipemic disorders and some risk factors associated with them (obesity, hypertension and alcoholism), in a representative sample of the population of 20 years of age and over in a locality typical of the peripheral zone of the Greater S. Paulo Region, Brazil, both in terms of the poverty of the population and with regard to the lack of public sources such as sewage, transport and housing, is undertaken. The following results were obtained: a) the prevalence of one risk factor was of about $55 \%$, and of two or more associated risk factors was of approximately $9 \%$ in the age group from 20 to 39 . There was found to be a prevalence of about $51 \%$ and $57 \%$, respectively of two or more associated risk factors for the age groups from 40 to 59 and 60 years of age and over; b) the prevalence of lipemic disorders proper was of about $49 \%, 58 \%$ and $57 \%$ respectively, for the age groups of 20 to 39, 40 to 59 and 60 years of age and over. In these cases the most prevalent risk factors among men were alcoholism and hypertension, either isolatedly or associated with obesity, and among women the most prevalent was obesity, alone or associated with hypertension; c) the most prevalent lipemic disorder was due to HDL-cholesterol, mainly among the younger people. The prevalence lower-thannormal HDL-cholesterol, was of about $28 \%$ and of "other lipemic disorders" was of $22 \%$ in the age group between 20 and 39 years of age. For those between 40 and 59 years of age the prevalence of low HDL-cholesterol and "other lipemic disorders" was of about $20 \%$ and $38 \%$ respectively. Among those above 60 years of age, the prevalence of HDL-cholesterol below normal was of about $7 \%$ and "other lipemic disorders" was of aproximately $50 \%$; d) obesity whether isolated or associated, was the most significant with higher "prevalence risk ratio". The lipemic disorders and risk factors studied probably represent a severe public health problem for the population of the peripheral zone of the city of $\mathrm{S}$. Paulo.

KEYWORDS: Hyperlipidemia, occurrence. Lipoproteins, HDL-cholesterol, blood. Cholesterol, blood. Triglycerides, blood. Risk.

\section{REFERÊNCIAS BIBLIOGRÁFICAS}

1. ANDERSON, K.M. et al. Cholesterol and mortality 30 years follow-up from Framingham Study. J. Amer. med. Ass., 257:2176-80, 1987.

2. BRAY, G.A. et al. Apud REUNIÓN CONSULTIVA CONJUNTA FAO/OMS/UNU DE EXPERTOS SOBRE NECESSIDADES DE ENERGIA Y DE PROTEINAS, Roma, 1981. Informe. Ginebra, Organizacion Mundial de la Salud, 1985.(OMS - Série de Informes Técnicos 724).

3. BRENN, T. The Tromso heart study: alcoholic beverages and coronary risk factors. J. Epidem. Community Hlth, 40:249-59, 1986.

4. BJÖRNTORP, P. Classification of obese patients and complications related to the distribution of surplus fat. Amer. J. clin. Nutr., 45:1120-5, 1987.

5. CALLEGARI, S. et al. Indagine retrospectiva su 778 pazienti con infarto acuto: rapporti tra sesso, etá e quadro lipemico. Acta bio-med. "Ateneo Parmense", 57(5/6):141-53, 1986.

6. CAMACHO, T.C. et al. Alcohol sonsumption and mortality in Alameda Country. J. chron. Dis., 40:229-36, 1987.

7. CARLSON, L.A. \& BÖTTIGER, L.E. Serum triglicerides, to be or not to be a risk factor for ischaemic heart disease? Atherosclerosis, 39:287-91, 1981.

8. CATELLI, P.W. et al. Incidence of coronary heart disease and lipoprotein cholesterol levels: the Framingham Study. J. Amer. med. Ass., 256:2835-8, 1986

9. DE FRANK, R.S. A longitudinal investigation of the relationship among alcohol consumption, psychosocial factors and blood pressure. Psychosom. Med., 49:236-49, 1987.

10. DIMSDALE, E.J. et al. Suppressed anger and blood pressure: the effects of race, sex, social class, obesity and age. Psychosom. Med., 48:162-8, 1986.
11. ELLIOTT, P. et al. Diet, alcohol, body mass and social factors to blood pressure: the Caerphilly Heart Study. J. Epidem. Community Hlth, 41:37-43, 1987.

12. GARRISON, R.J. et al. Obesity and lipoprotein cholesterol in the Framingham Offspring Study. $M e$ tabolism, 20:1053-60, 1980.

13.GILL, S.G. et al. Stroke and alcohol consumption. New. Engl. J. Med., 315:1041-6, 1986.

14.GILLUM, R.F. The association of body fat distribution with hipertension, hipertensive heart disease, coronary heart disease, diabetes and cardiovascular risk factors in men and women aged $18-79$ years. $J$. chron. Dis., 40:421-8, 1987.

15.GOLDBOURD, V. High risk versus public health strategies in primary prevention of coronary heart disease. Amer. J. clin. Nutr., 45:1185-92, 1987.

16.HUBERT, H.B. The importance of obesity in the development of coronary risk factors and disease: the epidemiologic evidency. Ann. Rev. publ. Hlth, 7:493-502, 1986.

17.ISRAELSSON, B. Role of alcohol, glicose intolerance and obesity in hypetrigliceridemia. Atherosclerosis, 62:123-7, 1986.

18.KEINBAUM, D.G. et al. Epidemiologic research: principles of quantitative methods. Belmont, Calif., Lifetime Learning Publications, 1982.

19.KOTTKE, I.E. et al. Projected population effects of a nutritional, blood pressure intervention on death rates from cardiovascular disease. Ann. clin. Rev., 16:170-3, 1984 .

20.LAPIDUS, $\mathbf{L}$. et al. Dietary habits in relation to incidence of cardiovascular disease and death in women: a 12 year follow-up of participants in the population study of women in Gothenburg, Sweden. Amer. J. clin. Nutr., 44:444-8, 1986. 
21.LEWIS, R. et al. Reducing the risks of coronary heart disease in individuals and in the population. Lancet, 1:956-9, 1986.

22.McMAHON, S.W. \& MACDONALD, G.J. Antihipertensive treatment and plasma lipoprotein levels: the associations in data from a population study. Amer. J. Med., 80:40-7, 1986.

23.MARTIN, M.J. et al. Serum cholesterol, blood pressure, and mortality: implications from a cohort of 361662 men. Lancet, 2:933-6, 1986.

24.MARTINS, I.S. et al. Níveis lipêmicos e alguns fatores de risco de doenças cardiovasculares em uma população do Município de São Paulo, SP (Brasil). Rev. Saúde publ., S. Paulo, 23:26-38, 1989.

25. MERIANS, D.R. et al. Relationship of exercise, oral contraceptives of plasma lipids and lipoprotein cholesterol in young women. Amer. J. Med., 78: 913-9, 1985.

26. POTTER, J.F. \& BEEVERS, D.G. The possible mechanisms of alcohol associated hipertension. Ann. clin. Res., 16:97-102, 1987.

27. POCOCK, S.J. et al. High density lipoprotein cholesterol is not a major risk factor for ischaemic heart disease in British men. Brit. med. J., 292: 515-9, 1986.

28. REICHLEY, B.R. et al. Centralizated obesity and cardiovascular disease risk in Mexican Americans. Amer. J. Epidem., 125:373-86, 1988.

29. ROSENTHAL, M.B. Effects of a high-complex-carbohydrate, low-fat, low-cholesterol diet on levels of serum lipids and estradiol. Amer. J. Med., 78:23-7, 1985.
30. SAMUELSSON, O. et al. Cardiovascular morbidity in relation to change in blood pressure and serum cholesterol levels in treated hypertension. J. Amer. med. Ass., 258:1768-76, 1988.

31. SHIE, S.M. Plasma lipid and lipoprotein concentration in Chinese males with coronary artery disease with and without hypertension. Atherosclerosis, 67:49-55, 1987.

32. STAMLER, J. et al. Is the relationship between serum cholesterol and risk of premature death from coronary heart disease continuos and graded? Findings in 356222 primary screenes of the multiply risk factor intervention trial (MRFIT). J. Amer. med. Ass., 256:2823-8, 1986.

33. TAYLOR, O.G. et al. High - density - lipoprotein in protein-energy malnutrition. Brit. J. Nutr., 47:489-94, 1987.

34. TURNER, R.W.D. \& BALL, K.P. Coronary heart disease. The size and nature of the problem: the role of fats. Postgrad. Med., 56:538-47, 1980.

35. WILLIAMS, P.T. et al. Associations of dietary fat, regional adiposity, and blood pressure in men. J. Amer. med. Ass., 257:3251-6, 1987.

36. WOLF, P.A. Cigarretes, alcohol and stroke. New Engl. J. Med., 315:1087-8, 1986.

Recebido para publicaçđo em 22/12/88 Reapresentado em 23/5/89

Aprovado para publicaçāo em 19/5/89 